\title{
Die strikt überwachte Chemotherapie der Tuberkulose - vor über 30 Jahren erstmals in Deutschland ambulant in größerem Umfang durchgeführt
}

\author{
Strictly Controlled Chemotherapy of Tuberculosis - Executed for the First Time \\ on a Larger Scale in Germany 30 Years Ago
}

\begin{abstract}
Als sich die antituberkulöse Chemotherapie als Therapie der Wahl erwiesen und anfangs der 60er-Jahre des letzten Jahrhunderts nach und nach durchgesetzt hatte, trat bald eine Reihe von praktischen Problemen stärker in den Vordergrund. Eines betraf die lange Dauer der Medikamenten-Einnahme (damals 18 bis 24 Monate). Mit dieser Zeitspanne, wesentlich länger als heutigentags erforderlich und durch die geringere Wirksamkeit der damals verfügbaren Medikamente bedingt, hatten manche Patienten große Schwierigkeiten. Derart lange Medikamentengaben waren unbekannt und ungewohnt, sonst unüblich; hinzu kam die Furcht vor den Nebenwirkungen der Behandlung, welche auch nicht selten in Erscheinung traten.
\end{abstract}

Zur damaligen Zeit war eine Kooperation der Patienten somit sicherlich noch schwerer zu erreichen als jetzt: Die Dauer der medikamentösen Therapie, ihre Nebenwirkungen bei längerfristiger Gabe von Mehrfachkombinationen und die schlechtere Verträglichkeit auch der einzelnen Komponenten führten viel häufiger dazu, dass der Patient die Therapie abbrach, weil er sich subjektiv bald besser fühlte; damit wurde es schwieriger, die weitere Behandlung einzusehen und zu akzeptieren, zumal die verbleibenden Beschwerden lediglich durch die Medikamente bedingt waren beziehungsweise auf diese bezogen wurden.

Diese Patienten, welche die Therapie vorzeitig abbrachen, wurden als „unzuverlässig“ eingeordnet. $\mathrm{Zu}$ ihnen gehörten natürlich auch diejenigen, welche prinzipiell unfähig und/oder nicht willens waren, die Medikamente langfristig in eigener Regie verlässlich und regelmäßig einzunehmen.

Um auch diesen Patienten eine Heilungschance zu eröffnen, wurde mancherorts eine kontrollierte Behandlung angeboten, vorwiegend während eines stationären Aufenthaltes. Das heißt, dass diese Patienten zustimmten, ihre Medikamente unter Aufsicht, „strikt überwacht“, einzunehmen.
Der erste Bericht über Erfahrungen in Deutschland mit dieser Behandlungsmöglichkeit während einer ambulanten [1,2] Tuberkulosetherapie wurde 1973 von mir publiziert [3]. Es handelte sich um 41 Patienten aus den Jahren 1968 - 1972, welche an einer infektiösen Lungentuberkulose litten und aus disziplinarischen Gründen mehrfach eine Klinik verlassen mussten oder den stationären Aufenthalt eigenmächtig abgebrochen hatten. Unter ihnen waren vor allem Alkoholiker [4] und dissoziale Menschen (Debile, Rauschgiftsüchtige, völlig Haltlose, Obdachlose und Kriminelle), an denen Kliniken und Gesundheitsbehörden gescheitert waren.

Als Kriterien für die Aufnahme in diese Studie „ambulante überwachte Chemotherapie“ galten:

- eine mindestens zweimalige disziplinarische und/oder eigenmächtige Beendigung des Klinikaufenthaltes,

- wiederholte inkorrekte Medikamenteneinnahme während der ambulanten Behandlung; dies ließ sich durch die Berechnung des Zeitraumes, für den die verordneten Medikamente nur hätten ausreichen müssen, erkennen,

- eindeutiges Rezidiv unter einer der Bakteriensensibilität angepassten und korrekt verordneten Therapie,

- und/oder eine schon aus früherer Behandlung bekannte mangelhafte Kooperation des Patienten.

Die Einwilligung des Patienten zu einem solchen Vorgehen zu erlangen war nicht schwierig. Denn dadurch entging er disziplinarischen Maßnahmen, wie Zwangsunterbringung (bei offener Tuberkulose) oder Sperrung der Sozialunterstützungen. Schwieriger war es schon, die Einwilligung der Kostenträger zu erhalten. Bedeutete doch eine strikt überwachte ambulante Therapie, dass die Patienten täglich (fünfmal in der Woche) die Praxis aufsuchen mussten. Und das überschritt im Allgemeinen das Budget des Kassenarztes. Aber obwohl diese Form der ambulanten Behandlung bisher in Deutschland noch nicht durchgeführt wurde,

Rubrikherausgeber: R. Kropp (federführend), U. Costabel, H. S. Fuchs, H. Jungbluth, H. J. Klippe, N. Konietzko, R. Loddenkemper, G. Neumann, M. Teschner

Korrespondenzadresse Dr. med. Ingeborg Schütz • Wohnstift Karlsruhe, App. I-4/9 • Erlenweg 2 - 76199 Karlsruhe 
waren die Krankenkassen von dieser Maßnahme zu überzeugen; denn eine ambulante Behandlung war ja weniger kostenaufwändig als ein monatelanger stationärer Aufenthalt. Schließlich haben später die Ergebnisse die Maßnahmen gerechtfertigt.

Über $60 \%$ der behandelten Patienten waren Alkoholiker. Über die Hälfte war ledig oder geschieden. Die überwiegende Zahl der Patienten war ungelernter Arbeiter oder Maurer. Aber auch vier Patienten aus Krankenpflegeberufen waren darunter.

Die in die Studie aufgenommenen Patienten mussten arbeitstäglich (fünfmal wöchentlich) die lungenfachärztliche Praxis ${ }^{1}$ aufsuchen. Hier erhielten sie ihre Medikamente (in einmal täglicher Gabe) als intravenöse Infusion, intramuskuläre Injektion beziehungsweise oral. Letztere wurden unter der Aufsicht einer erfahrenen Sprechstundenhilfe eingenommen. Auch wurden regelmäßig die erforderlichen Kontrolluntersuchungen vorgenommen [5].

Patienten, die während dieser Therapie auch nicht kooperierten², brachen diese vorwiegend bereits innerhalb des ersten Monats nach Beginn der überwachten Behandlung ab. Wurde der erste Monat aber durchgehalten, dann kamen die Patienten auch im ganzen ersten Jahr regelmäßig oder mit nur kurzen Unterbrechungen. Wenn hierdurch auch ein Rezidiv nicht zu erwarten war, zog sich doch die überwachte Therapie durch solche Unterbrechungen länger hin als gewöhnlich, wobei zu bedenken ist, dass zur damaligen Zeit die Chemotherapie ohnehin länger durchgeführt werden musste als jetzt.

Bei der Überprüfung, welche Patienten am kooperativsten waren, stellte sich heraus, dass Alkoholiker im Allgemeinen als kooperativ angesehen werden konnten, selbst wenn sie schon zerebrale Folgeschäden hatten. Das Gleiche gilt auch für die Gruppe der im Krankenhaus Uneinsichtigen. In der Gruppe der Dissozialen war dagegen die überwachte Chemotherapie schlecht praktikabel. Hier hätte man nur mit einem gewissen Erfolg rechnen können, wenn man die Medikamente den Patienten regelmäßig in die Wohnung hätte bringen können.

Bei manchen Patienten waren die Beendigung der Injektions- beziehungsweise Infusionsbehandlung oder die Wiederaufnahme der Arbeit Ursache für ihren Therapieabbruch. Diese Zahl lag im geschilderten Kollektiv relativ hoch im Gegensatz zu Berichten aus anderen Ländern. Das erklärt sich daraus, dass sich dort Organisationen mit Psychologen, Fürsorgerinnen und Schwestern der Patienten annehmen, und dass oft auch noch gewisse Möglichkeiten des Zwanges bestehen.

Von den 42 Patienten war die überwachte ambulante Therapie bei ${ }^{2} / 3$ erfolgreich. Bei diesen Kranken konnte sie über die individuell erforderliche Zeit durchgehalten werden. Die Tuberkulose besserte sich klinisch und röntgenologisch, eine dauerhafte Sputumkonversion konnte erreicht werden.

\section{Postskriptum}

Vergleicht man das geschilderte Vorgehen mit heutigen Verhältnissen, vor allem mit der Propagierung von DOT (directly observed treatment) [6] durch die Weltgesundheitsorganisation (WHO), so ist letztere ausgefeilter, weltweit angewandt und auf einen erheblich größeren Patientenkreis bezogen. Doch klingen die der Publikation von 1973 zugrunde liegenden Verhältnisse, Überlegungen und Vorgehensweisen, auch die Ergebnisse, wie ein Vorgriff auf diese derzeit erfolgreiche Strategie (DOT), welche gleichfalls eine „strikt überwachte“ Chemotherapie als ein wesentliches Element ihres Vorgehens zum Inhalt hat [7].

\section{Literatur}

${ }^{1}$ Eddison GG, Farmer BM. A control study of home versus hospital treatment of tuberculosis; three-year study results. Amer Rev resp Dis 1971; 103: 886-887

2 Onstad GD, Sbarbaro JA, Rotenberg L. Posthospital chemotherapy of the unreliable patient. Amer Rev Resp Dis 1970; 101: 258-264

${ }^{3}$ Schütz I. Ambulante Chemotherapie der Lungentuberkulose unter besonderer Berücksichtigung der strikt überwachten, intermittierenden Medikamentengabe. Prax Pneumol 1973; 27: 154-168

${ }^{4}$ Kerntke G. Tuberkulose und Alkohol. Prax Pneumol 1968; 22: $349-367$

${ }^{5}$ Radenbach KL. Überwachung und Kontrolluntersuchungen bei ambulanter antituberkulöser Behandlung. Prax Pneumol 1965; 19: $145-155$

${ }^{6}$ World Health Organization. Framework for effective tuberculosis control: WHO/TB/94.179. Genf: WHO, 1994

${ }^{7}$ World Health Organization. What is DOTS? A guide to understanding the WHO-recommended TB control strategy known as DOTS. WHO/ CDS/CPC/TB/99.270. Genf: WHO, 1999

\footnotetext{
${ }^{1}$ Die ambulante überwachte Therapie wurde in meiner Praxis in BerlinMoabit durchgeführt. Gleichzeitig war ich als Oberärztin an der Lungenklinik Heckeshorn bei Herrn Professor Radenbach tätig.

2 Anstelle des Begriffs „Kooperation“ ist heute „Compliance“ bzw. „Adherence“" üblich.
} 